Polymer Chemistry

and L iposome Technology

David A. Tirre11

Polymer Science and Engineering Department

University of Massachusetts

Amherst, MA 01003

Polymer chemistry has a great deal to offer in the construction of synthetic liposomal membranes for use in biology and medicine. This chapter explores the preparation and properties of polymeric liposomes, with particular emphasis on the use of controlled polyelectrolyte adsorption to manipulate 1iposomal membrane properties.

Nature has combined the disciplines of polymer chemistry and colloid science to remarkable effect in her design of the bilayer membranes that surround cells and subcellular organelles. These membranes do many intriguing things: they recognize one another, they respond to the binding of drugs and hormones, and they control the flow of mass, information and energy within and between cells. It would be natural, then, for us to select such structures as we try to intervene in biological processes through the controlled delivery of drugs, markers and genetic material. Following Bangham's discovery (1) of the barrier properties of lipid vesicles, or liposomes, there has grown an enormous literature devoted to liposomal delivery (2-6). What has emerged more recently is the realization that polymer chemistry has much to offer in the technological development of liposomal delivery systems.

Research on liposomal delivery systems has been motivated in large part by the notion that the material to be delivered can be entrapped in the liposomal interior, and that the entrapped material will remain inside until the liposome is opened at its target. Implementation of this idea requires the formulation of riposomal systems that are resistant to leakage of entrapped substances, resistant to attack by foreign agents used in the preparation of the delivery system (e.g. surfactants and organic solvents), stable toward attack by endogenous agents (e.g. enzymes or plasma proteins) and capable of tissue localization or selective rupture at the target. The following discussion will provide an overview of the present state of the union between polymer chemistry and liposome technology. We will address first the methods of preparation of 
polymeric liposomal systems, and then proceed to an evaluation of the contributions that polymer chemistry can make to the solution of the problems outlined above.

\section{Preparative Methods}

The major approaches to the preparation of polymeric 1iposomal systems may be distinguished on the basis of the locus of polymerization. Most effort to date has been directed toward the preparation of phospholipid analogues that are intrinsically polymerizable through reactions of attached vinyl, butadienyl, diacetylenic, isocyano, thiol or disulfide functions. This approach produces liposomes in which the primary permeability barrier - the bilayer itself - is polymeric. The second approach invokes the attachment of extrinsic macromolecules to the 1ipid bilayer, in a fashion analogous to the assembly of biological membranes.

Polymerizable Lipids. The preparation of polymerizable lipids has been extensive1y reviewed (7-10), so the present discussion will be brief. It is useful to distinguish two portions of amphiphilic, membrane-forming lipids: a polar headgroup that forms the interface between the membrane and its environment, and the hydrocarbon chains that provide the primary barrier to the escape of entrapped solutes. It is important in the design of polymerized liposornes that one keep in mind these distinct functions, and that the locus of polymerization be selected in such a way that the desired functional properties are preserved or enhanced. Polymerization through functional groups appended to the lipid headgroup would be expected to preserve the packing properties of the hydrocarbon chains (assuming that the geometric requirements of the polymerization are consistent with those of chain packing), but would alter the surface recognition behavior of the membrane. On the other hand, polymerization in the hydrocarbon core - either in the middle of the chain or at its end - offers the prospect of an unperturbed lipid surface, but compromises in a profound way the packing of the hydrocarbon chains. It is important to point out, however, that such changes in packing may be advantageous, in that the barrier properties of the bilayer may be significantly enhanced by polymerization. An example of reduced solute leakage from polymerized liposomes will be discussed in the following section.

Attachment of Extrinsic Macromolecules. Attachment of synthetic polymers to the Tiposomal surface has been accomplished by at least four different routes (Scheme I). The simplest route - but also that least amenable to control - involves the adsorption of unmodified polymer chains (11). In adopting this route, one must walk a fine line between systems in which the polymer-bilayer interactions are too weak to maintain a substantial surface concentration of chains, and systems in which those interactions are strong enough to cause vesicle reorganization and lysis. A second route - with distinct advantages and well established biological precedent - is the use of hydrophobic "anchoring groups" to hold in place even weakly bound polymer chains. Suitable anchors are single or double chain surfactants (12:13) or cholesterol (14), and in principle such anchors 
may be introduced first either to the polymer chain (Scheme I, route 2 ) or to the vesicle surface (Scheme I, route 3 ). The biological analogy of course is the widespread occurrence of hydrophobic peptide sequences in membrane-bound proteins, which serve a similar anchoring function. Finally, both Regen (15) and Ringsdorf (16) have suggested that the formation of liposomes from surfactants bearing polymerizable counterions might be followed by polymerization within the double layer to provide a surface coating of polyelectrolyte chains (Scheme I, route 4).

The reader should also be aware of a very substantial, related body of work that addresses the anchoring of extrinsic, naturally occurring macromolecules to 1iposomal surfaces $(2-6,17)$. Because this work has already been extensively reviewed, the present chapter will not discuss this very interesting and important subject.

\section{Properties of Polymeric Liposomes}

Liposomes from Polymerizable Lipids. Again the discussion will be brief, and the reader is directed to the several excellent reviews of polymerized vesicles (3). We discuss here only one very recent example of the preparation and properties of such a system. This example has much to recommend it, and is described by the original authors as, "the polynierizable lipid of choice for a wide variety of mechanistic and practical applications."

Regen and coworkers described in 1986 the preparation and polymerization of 1,2-bis[12(1 ipoyloxy)dodecanoyl]-sn-glycero3-phosphocholine 1 (18). Aqueous dispersions of 1 produced by

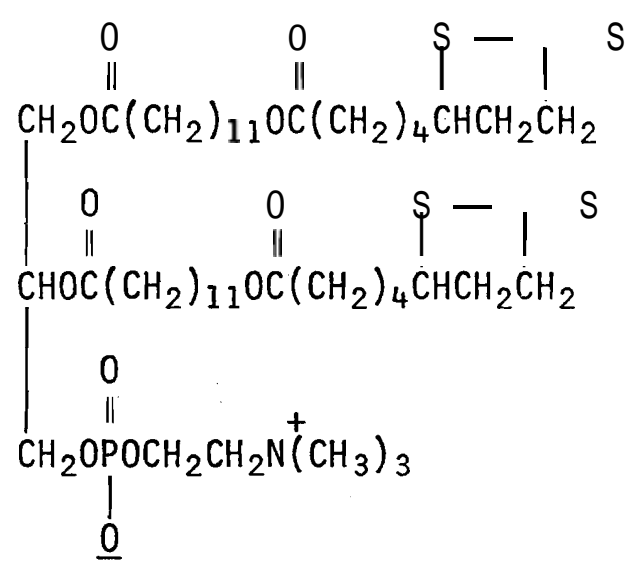

injection of ethanolic solutions were shown to consist of vesicles of average diameter 270-400A. Treatment of such dispersions with $10 \mathrm{~mol} \%$ dithiothreitol (DTT) for $4 \mathrm{hr}$ at $27^{\circ} \mathrm{C}$ produced complete polymerization of 1 , as shown by thin layer chromatographic analysis for unreacted monomer and by the disappearance of the characteristic UV absorption maximum of the monomer at $333 \mathrm{~nm}$. Polymerization was accompanied by a small decrease in particle size as reported by dynamic light scattering, but the presence of closed vesicles was suggested by electron microscopy and confirmed by solute entrapment. The polymerized vesicles were stable to lysis even in $1 \%$ solutions of sodium dodecyl sulfate (SDS) at $60^{\circ} \mathrm{C}$; in contrast, vesicles of monomeric 1 were destroyed in $0.05 \%$ SDS a t room temperature. The 
size distribution of the polymerized vesicle dispersion was shown to be stable for at least 3 months at room temperature, and the dispersion retained $61 \%$ of its entrapped sucrose after $4 \mathrm{hr}$ at $23^{\circ} \mathrm{C}$, as compared to $32 \%$ retention by monomeric 1 under identical conditions.

This system indeed has much to recommend it. The polymerization conditions are remarkably mild, and appear to confer on the bilayer increased barrier properties and improved resistance to detergent Iysis. In addition, the construction of the polymer chain by the formation of disulfide bonds leaves open the prospect of depolymerization in a reducing biological environment and subsequent degradation.

Liposomes Bearing Extrinsic Macromolecules. This section will consider the properties of liposomes to which extrinsic macromolecules are bound by adsorption or by hydrophobic anchors. Regen (15) and Ringsdorf ( 16$)$ have discussed the consequences of polymerization of surfactant counterions (9).

Liposomes Bearing Adsorbed Chains. Much of the work to date on liposomes'bearing adsorbed chains has concerned the $\mathrm{pH}$-dependent adsorption of acidic polyelectrolytes on liposomes prepared from phosphatidylcholines $(11,19-21)$. Interest in this problem has it s origin in the $\mathrm{pH}$-dependent conformational and solubility properties of acidic polyelectrolytes. One might expect, for example, that a poly (carboxylic acid) would be converted upon acidification from a charged, hydrophilic structure to a globular, hydrophobic coil, poorly solvated by aqueous media. The liposomal surface provides a place of refuge for the hydrophobic chain; the chain in turn alters in a profound way the geometric and thermodynamic factors that combine to determine bilayer structure. One can easily imagine that the polymer-phospholipid mixture might adopt a totally different aggregate morphology, and that in the course of the structural reorganization the barrier properties of the bilayer would be lost. Scheme II shows a reasonable working hypothesis, in which proton-driven polyelectrolyte adsorption induces a vesicle-to-mice1le transition upon acidification (22).

Scheme II suggests that polyelectrolyte adsorption might provide a means of preparing $\mathrm{pH}$-dependent liposomal delivery systems. Given the variations in $\mathrm{pH}$ that characterize certain pathological states (23) and certain subcellular organelles (24), the ability to a1ter membrane properties in a controlled, pH-dependent manner is a powerful methodology. Indeed, it has become clear that the cell uses subtle changes in $\mathrm{pH}$ to control its intracellular processing of ligands and receptors (24), and that infectious microorganisms have developed mechanisms to exploit these $\mathrm{pH}$ changes to gain entry to the cytoplasm (25).

Most of our work to date has concerned the interactions of poly(acrylic acid) derivatives with vesicle membranes prepared from phosphatidylcholines. In particular, poly(2-ethylacrylic acid)

(PEAA, 2) is a hydrophobic poly(carboxylic acid) that undergoes a conforrnational transition of the kind described above (26-28). That this conformational transition occurs near neutral pH makes PEAA a candidate for use in $\mathrm{pH}$-dependent 1iposomal delivery systems i $n$ biology and medicine. 
$\mathrm{CH}_{2} \mathrm{CH}_{3}$

$\mathrm{CO}_{2} \mathrm{H}$

Figure 1 shows that PEAA indeed functions well in this role $(19)$. The Figure shows the efflux of the fluorescent dye carboxyfluorescein from unilaniellar vesicles of egg yolk phosphatidylcholine suspended in an aqueous solution of PEAA. Escape of the dye is very slow at pH 7.4, but essentially instantaneous upon acidification of the suspension to $\mathrm{pH} 6.5$. We have since demonstrated the $\mathrm{pH}$-triggered release of other substances from phosphatidylcholine vesicles by this method, and the technique should be completely general in its application to the controlled release of water soluble compounds.

Additional uses of these and related systems are suggested by the fact that many enzymes are known to yield acidic products via oxidative or hydrolytic reactions. Glucose oxidase, for example, catalyzes the oxidation of glucose to gluconic acid, which is a suitable source of $\mathrm{H}^{+}$. A phosphatidylcholine suspension containing both glucose oxidase and PEAA should then be sensitive to glucose concentration, in that increasing glucose concentrations should lead to vesicle rupture with release of contents. Such systems might prove to be useful in self-regulated insulin delivery or in monitoring of glucose concentrations in physiologic fluids.

Figure 2 demonstrates the viability of this approach to the preparation of glucose-sensitive membranes (20). The Figure shows the results of an experiment in which dilauroy phosphatidylcholine was suspended in an unbuffered, aqueous solution of PEAA and glucose oxidase at pH 7.4. Upon addition of glucose, the optical density of the solution was rapidly reduced, and reached a value less than $10 \%$ of the original after approximately $30 \mathrm{~min}$. The reduction in optical density signals a reorganization of the bilayer that must be analogous to that induced by direct addition of $\mathrm{H}^{+}$as in Figure 7 , so that we would anticipate quantitative release of vesicle contents under the conditions of Figure 2. This system is of interest not only from the point of view of its potential applications in diagnosis and therapeutics, but also by virtue of its analogy to the "second messenger" signalling processes so important in cell biology. That is, the signal is initiated by a rise in the concentration of glucose, but it is a second substance $\left(\mathrm{H}^{+}\right)$that carries the message to the effector molecule (PEAA). The analogy to hormonal second messenger systems is crude, but quite real.

Liposomes Bearing Anchored Chains. We have very recently extended our work on the PEAATphosphatidylcholine system by immobilizing PEAA on the surface of egg lecithin via a hydrophobic anchor (Maeda, M.; Kumano, A.; Tirrell, D.A. Preprints ACS Div. Polym. Chem., in press). The method involves the coupling of thiolated PEAA to the maleimido phospholipid 3, which was incorporated at a level of ca. $10 \%$ into preformed lecithin vesicles. Michael addition of the polymer-bound thiol groups to the $\mathrm{N}$-alkylmaleimide functions on the vesicle surface results in immobilization of 50-60 $\mathrm{\mu g}$ of PEAA per mg of lipid. Following fractionation of the sample by size exclusion 
chromatography, acidification of the vesicle fractions causes rapid release of vesicle contents. These results demonstrate that it is indeed possible to anchor sufficient PEAA to effect useful changes i n membrane structure and function.

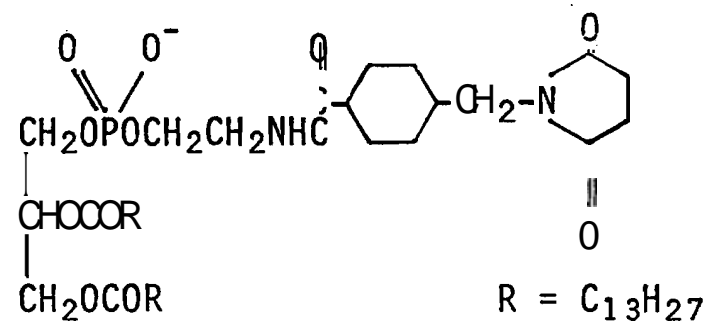

Conclusions

Polymer chemistry offers a number of powerful strategies for the controlled manipulation of the properties of 1iposomal membranes. Polymerization within the bilayer can provide membranes of improved stability and barrier properties. Controlled adsorption or anchoring of polyelectrolytes can render the liposomal membrane sensitive to chemical and physical signals, and so can afford 1 iposomal delivery systems susceptible to selective rupture at predetermined physiological targets or under predetermined pathological conditions.

$\underline{\text { Literature Cited }}$

1. Bangham, A.D.; Standish, M.M.; Watkins, J.C. J. Mol. Biol. 1965, 13., 238.

2. Gregoriadis, G., ed. Liposome Technology; CRC Press: Boca Raton, 1984.

3. Ostro, M.J., ed. Liposomes; Marcel Dekker: New York, 1983.

4. Bangham, A.D., ed. Liposome Letters; Academic Press: New York, 1983.

5. Knight. C.G.. ed. Liposomes: From Physical Structure to Therapeutic Applications; Elsevier: Amsterdam; 1981.

6. Gregoriadis, G.; Senior, J.; Trouet, A., eds. Targeting of Drugs; Plenum: New York, 1982.

7. Fend7er, J.H. Membrane Mimetic Chemistry; Wiley: New York, 1982.

8. Tirrell, D.A. ; Donaruma, L.G. ; Turek, A.B., eds. Macromolecules as Drugs and as Carriers for Biologically Active Materials; N.Y. Acad. Sci.: New York, 1985.

9. Bader, H.; Dorn, K.; Hupfer, B.; Ringsdorf, H. Adv. Polym. Sci. 1985, 64, 1.

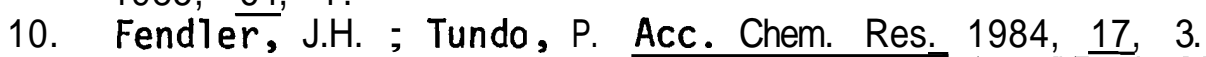

11. Seki, K.; Tirrell, D.A. Macromolecules T984, 17, 1692.

12. Sunamoto, J.; Iwamoto, K.; Takada, M.; Yuzuriha, T.; Katayama, K. I n Recent Advances i $n$ Drug Delivery Systems; Anderson, J.M. ; Kim, S.W., eds. Plenum: New York, 1984, p. 153.

13. Martin, F. J. ; Papahadjopoulos, D. J. Biol. Chem. 1982, 257, 286.

14. Ottenbrite, R.M.; Sunamoto, J.; Sato, T.; Oka, M. Prepr. ACS Div. Polym. Chem. 1985, 26(1), 212.

15. Fukuda, H.; Diem, T.; Stefely, J.; Kezdy, F.J.; Regen, S.L. L Am. Chem. Soc. 1986, 108, 2321. 
16. Aliev, K.V.; Ringsdorf, H.; Schlarb, B.; Leister, K.H. Makromol. Chem. Rapid Commun. 1984, 5, 345.

17. Sunamoto, J.; Iwamoto, K. CRC Crit. Revs. Ther. Drug Carrier . Systems 1986, 2, 117.

18. Sadownik, A. ; Stefely, J. ; Regen, S. L. J. Am. Chem. Soc. 1986, 108, 7789.

19. Tirrell, D.A. ; Takigawa, D.Y. ; Seki, K. Ann. N.Y. Acad. Sci. 1985, 446, 237.

20. Devlin, B.P.; Tirrell, D.A. Macromolecules 1986, 19, 2465.

21. Borden, K.A.; Eum, K.M.; Langley, K.H.; Tirrell, D.A. Macromolecules 1987, 20, 454.

22. Takigawa, D.Y. ; Tirre $\overline{11}$, D.A. Makromol. Chem. Rapid Commun. $1985,6,653$.

23. Yatvin, M.B. ; Kreutz, W.; Horwitz, B.A.; Shinitsky, M. Science 1986, 210, 1253.

24. Yamashiro, D.J.; Tycko, B.; Fluss, S.R.; Maxfield, F.R. Cell, 1984, 37, 789.

25. White, J.; Kielian, M.; Helenius, A. Quart. Revs. Biophys. 1983, $16,151$.

26. Fichtner, F.; Schonert, H. Colloid Polym. Sci. 1977, 255, 230.

27. Joyce, D.E.; Kurucsev, T. Polymer 1981, 22, 415.

28. Sugai, S., Nitta, K. Ohno, N. ; Nakano, H. Colloid Polym. Sci. $1983,261,159$. 
Figure 1. Efflux of carboxyfluorescein from sonicated egg yolk phosphatidylcholine vesicles suspended in $50 \mathrm{mM}$ Tris $-\mathrm{HCl}, 100 \mathrm{mM}$ $\mathrm{NaCl}$ at indicated $\mathrm{pH}$.

Figure 2. Optical density of a multilamellar suspension of DLPC in an aqueous solution of PEAA and glucose oxidase, prior and subsequent to addition of glucose. 


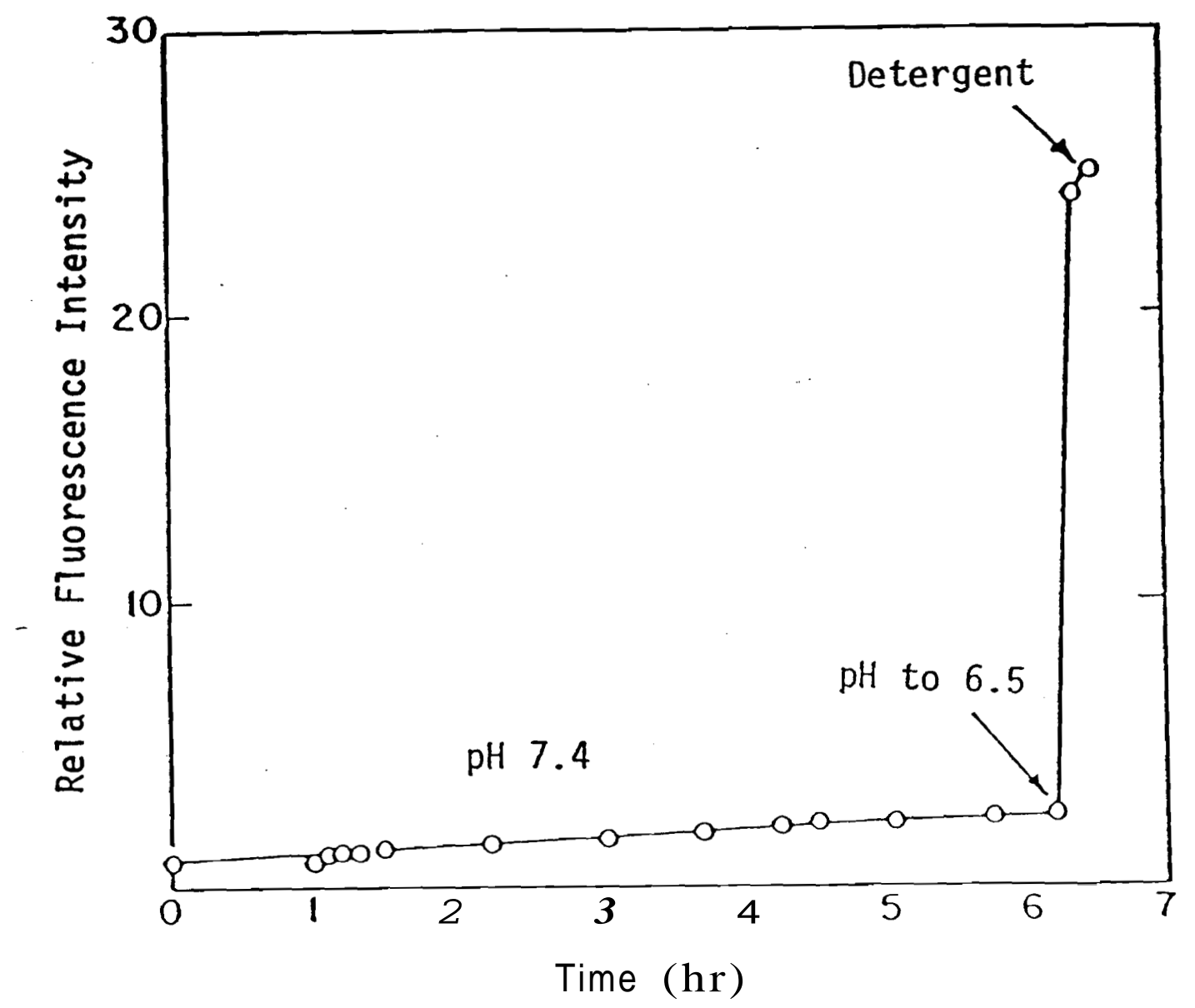




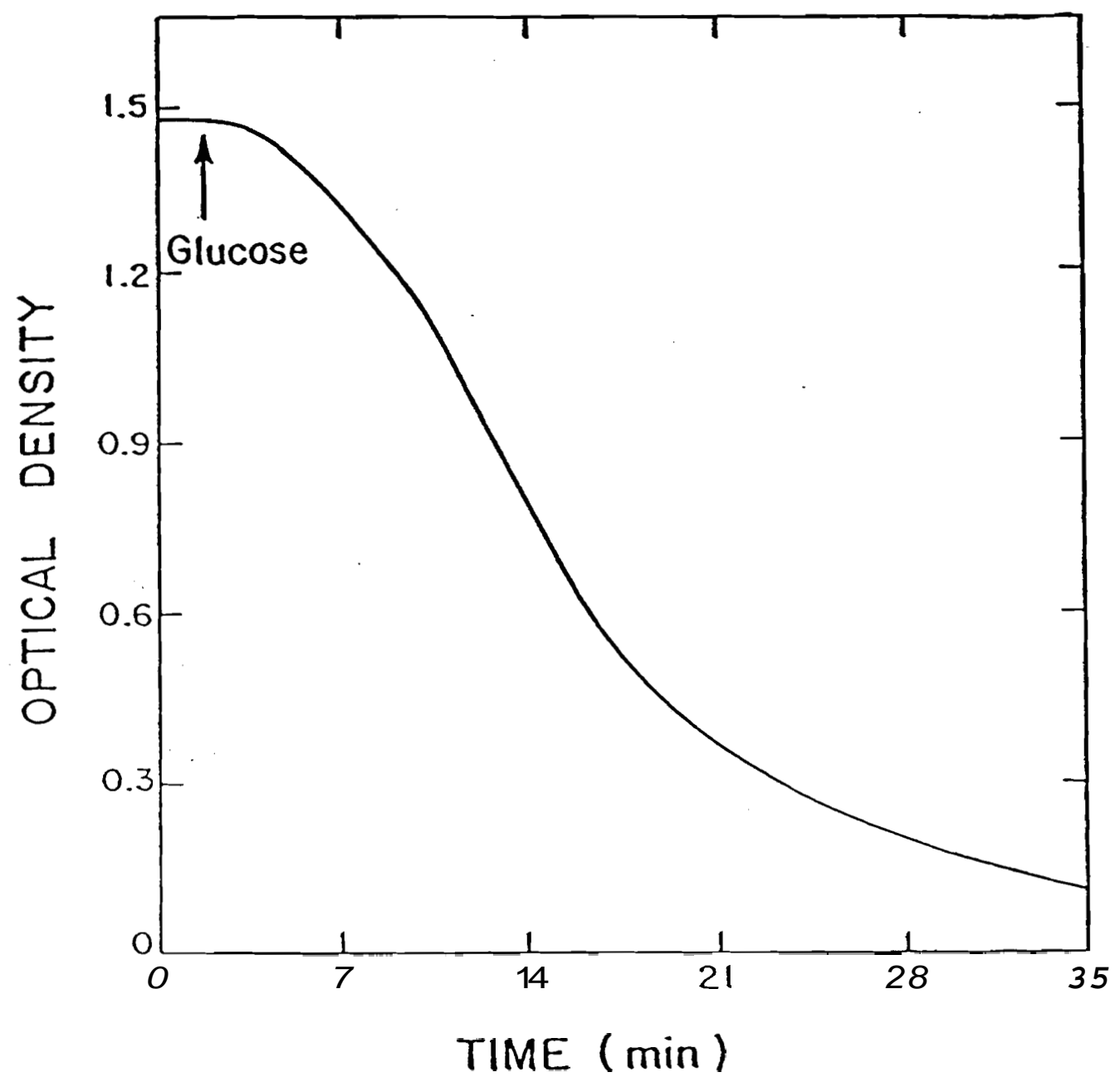



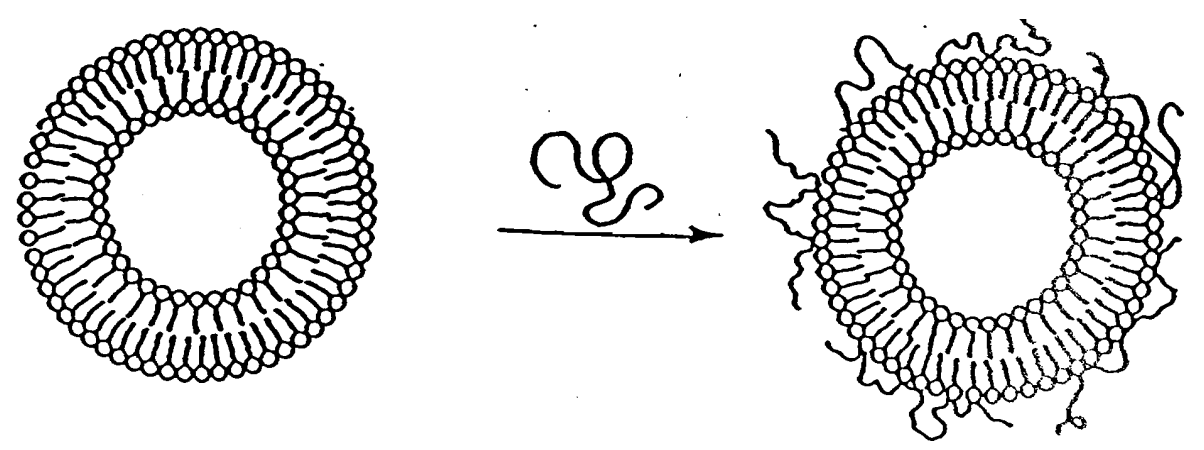

1
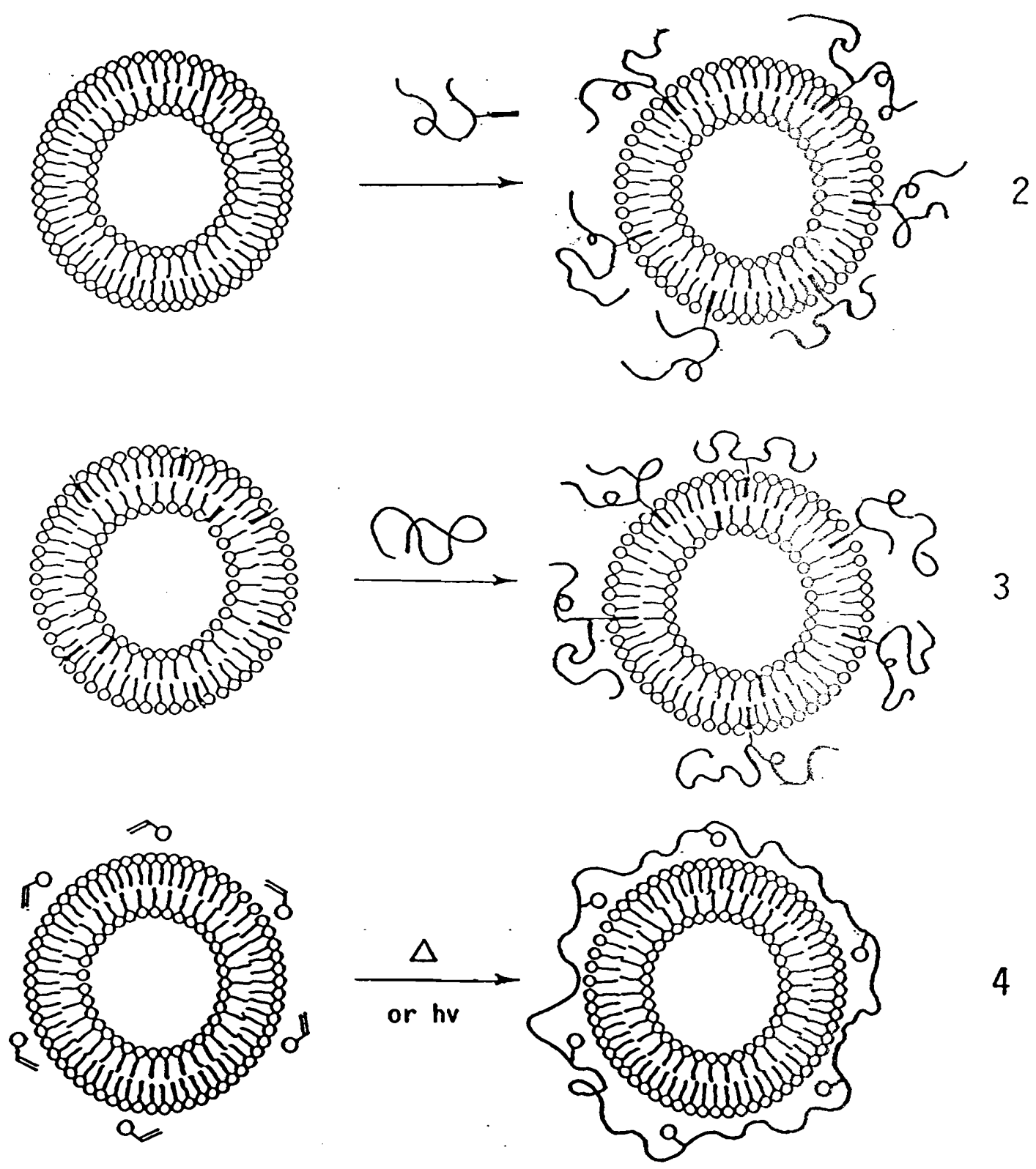


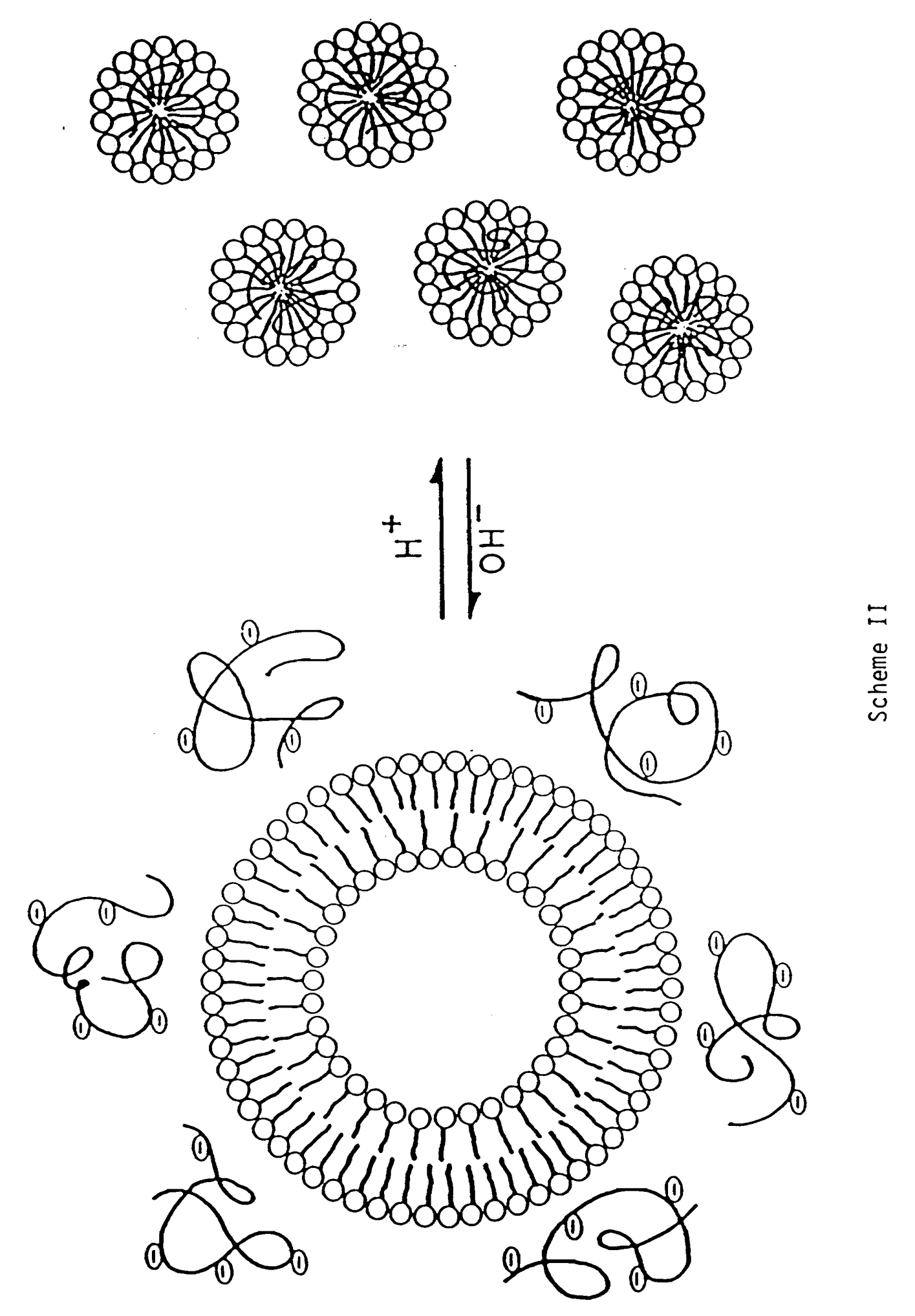

